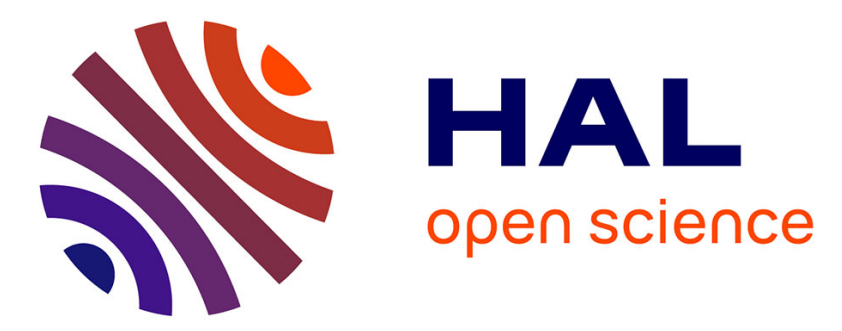

\title{
Magnetic resonance imaging for diagnosis of early Alzheimer's disease
}

Olivier Colliot, Lorraine Hamelin, Marie Sarazin

\section{To cite this version:}

Olivier Colliot, Lorraine Hamelin, Marie Sarazin. Magnetic resonance imaging for diagnosis of early Alzheimer's disease. Revue Neurologique, 2013, 169 (10), pp.724-8. 10.1016/j.neurol.2013.07.013 . hal-00867481

\section{HAL Id: hal-00867481 https://hal.inria.fr/hal-00867481}

Submitted on 16 Oct 2017

HAL is a multi-disciplinary open access archive for the deposit and dissemination of scientific research documents, whether they are published or not. The documents may come from teaching and research institutions in France or abroad, or from public or private research centers.
L'archive ouverte pluridisciplinaire HAL, est destinée au dépôt et à la diffusion de documents scientifiques de niveau recherche, publiés ou non, émanant des établissements d'enseignement et de recherche français ou étrangers, des laboratoires publics ou privés. 


\section{L’imagerie par résonance magnétique pour le diagnostic précoce de la maladie d'Alzheimer}

\section{Magnetic resonance imaging for diagnosis of early Alzheimer's disease}

Olivier Colliot ${ }^{1,2,3,4,5}$, Lorraine Hamelin ${ }^{1,2,3,4,5,6}$, Marie Sarazin ${ }^{7}$

${ }^{1}$ Université Pierre et Marie Curie-Paris6, Centre de Recherche de l'Institut du Cerveau et de la Moelle épinière, UMR-S975, Paris, France

${ }^{2}$ Inserm, U975, Paris, France

${ }^{3}$ CNRS, UMR 7225, Paris, France

${ }^{4}$ ICM - Institut du Cerveau et de la Moelle épinière, Paris, France

${ }^{5}$ INRIA, Centre Paris-Rocquencourt, France

${ }^{6}$ Department of Neurology, Institut de la Mémoire et de la Maladie d'Alzheimer,- IM2A, Groupe Hospitalier Pitié-Salpêtrière, Paris, France

${ }^{7}$ Université Paris Descartes, Sorbonne Paris Cité, INSERM UMR S894, Service de Neurologie, Hôpital Sainte-Anne, Paris

\section{Correspondance:}

Olivier Colliot

Centre de Recherche de l’Institut du Cerveau et de la Moelle épinière - CRICM

Batiment ICM

Hôpital de la Pitié-Salpêtrière

47, boulevard de l'hôpital

75013 Paris

France

Phone: +33 157274365

e-mail: olivier.colliot@upmc.fr 


\title{
L’imagerie par résonance magnétique pour le diagnostic précoce de la maladie d'Alzheimer
}

\section{Magnetic resonance imaging for diagnosis of early Alzheimer's disease}

\begin{abstract}
A major challenge for neuroimaging is to contribute to the early diagnosis of Alzheimer's disease (AD). In particular, magnetic resonance imaging (MRI) allows detecting different types of structural and functional abnormalities at an early stage of the pathology. Anatomical MRI is the most widely used technique and provides local and global measures of atrophy. The recent diagnostic criteria of “MCI due to AD” include hippocampal atrophy which is considered a marker of neuronal injury. Advanced image analysis techniques generate automatic and reproducible measures both in the hippocampus and throughout the wholebrain. Recent modalities such as diffusion-tensor imaging and resting-state functional MRI provide additional measures that could contribute to the early diagnosis but require further validation.
\end{abstract}

\section{Résumé}

Un des enjeux majeurs de la neuroimagerie est de contribuer au diagnostic précoce de la maladie d'Alzheimer. L’imagerie par résonance magnétique (IRM) permet de détecter différents types d'altérations structurelles et fonctionnelles dès les premiers stades de la maladie. L'IRM anatomique est la technique la plus répandue et fournit des mesures d’atrophie cérébrale locales ou régionales. Les nouveaux critères de diagnostic de la maladie d'Alzheimer au stade des troubles cognitifs légers incluent l'atrophie hippocampique, qui est alors considéré comme un marqueur d’atteinte neuronale. Les techniques avancées d'analyse d'images génèrent des mesures automatiques et reproductibles à la fois dans l'hippocampe et dans l'ensemble du cerveau. Des modalités récentes telles que l'imagerie du tenseur de diffusion ou l'IRM fonctionnelle de repos fournissent des mesures complémentaires qui pourraient également contribuer au diagnostic précoce mais nécessitent d’être plus largement validées.

Mots-clés : maladie d’Alzheimer ; imagerie par résonance magnétique ; IRM anatomique ; IRM fonctionnelle ; IRM de diffusion 


\section{Introduction}

The increasing global prevalence and societal cost of Alzheimer's disease (AD) explains the need for early diagnosis, in order to develop effective treatments able to stop the pathophysiological process. By integrating new methods for diagnosis based on both neuroimaging and biological markers, the diagnosis accuracy has improved, allowing diagnosis of MCI (mild cognitive impairment) due to AD. Among neuroimaging techniques, different types of magnetic resonance imaging (MRI) acquisitions may contribute to the early diagnosis of $\mathrm{AD}$ by detecting different structural and functional alterations. Anatomical MRI is the most widely used technique and allows measuring atrophy in various brain regions. Diffusion tensor imaging provides information on the integrity of white matter tracts. Functional MRI can be used to study alterations of functional connectivity.

\section{Anatomical Magnetic Resonance Imaging}

Besides its value to rule out others causes of cognitive dysfunction (normal pressure hydrocephalus, subdural hematoma e.g.), which remains an essential step in the clinical reasoning, MR imaging is now considered an essential part of $\mathrm{AD}$ diagnosis. In $\mathrm{AD}$, anatomical MRI allows studying the presence of atrophy along with possible signs of associated vascular pathology. Brain atrophy can be assessed with 3D T1-weighted MRI acquisitions with approximately $1 \mathrm{~mm}$ isotropic resolution. Anatomical MRI changes map accurately upstream to Braak stages of neurofibrillary tangle deposition and downstream to neuropsychological deficits. Neuropathologic studies in patients with AD showed that MRI alterations correlate with tau deposition, Braak stage, and neuronal counts (Vemuri et al. 2008). Moreover, hippocampal atrophy is correlated with CSF tau markers (de Souza et al. 2012) and with episodic memory deficit in AD patients (Sarazin et al. 2010).

\section{Medial Temporal Lobe atrophy (MTL)}

Hippocampal atrophy appears to be a robust biomarker of AD. Measurements of medial temporal lobe atrophy can distinguish AD from age-matched controls with overall sensitivity and specificity greater than $80 \%$ (Lehéricy et al. 2007). Medial temporal atrophy can be assessed in clinical routine by visual scales (Scheltens et al. 2002). Image segmentation methods have the advantage to provide automated and reproducible measures of hippocampal volume (Colliot et al. 2008). Hippocampal shape analysis has the potential to detect subtle alterations that are not reflected in global volumetric measurements (Gerardin et al. 2009). 
Hippocampal atrophy is not specific to Alzheimer's pathology and is described in other degenerative pathologies such as Parkinson disease (with or without dementia), vascular dementia (Laakso et al. 1996), dementia with Lewy Body (Hashimoto et al. 1998) and fronto temporal dementia (de Souza et al. 2013), or even in depression and “normal aging”. The overlap of hippocampal volume measures between $\mathrm{AD}$ and others medical conditions or aging limits its interpretation when considered without clinical data.

In longitudinal MRI studies, the annual rate of hippocampal atrophy is higher in AD (from 2,2\% to 5,9\%) than in normal aging (from 0,2\% to 1,7\%) (Lehéricy et al. 2007). Moreover, MCI patients with fast cognitive decline would present a higher hippocampal atrophy rate than the patients remaining stable and the controls (Jack et al. 2000).

The use of both MRI and biological tools could improve early diagnosis. For example, in a a cross-sectional study, the combination of CSF biomarkers and MRI provided better prediction than either source of data alone (Vemuri et al. 2009). Ultra-high field MRI, allowing in particular hippocampal subfield identification, is a promising research lead to increase the diagnostic accuracy of medial temporal measures (Mueller et al. 2011).

\section{Vascular component associated to AD}

Leucoaraiosis (white matter hyperintensities) and lacunar infarcts can be assessed using T2weighted and FLAIR (FLuid Attenuated Inversion Recovery) sequences. Varying degrees of small vessel disease, ischaemic-related white matter changes and one or more microinfarcts are frequently observed in patients presenting cognitive decline. T2*-weighted GRE sequences are used to study the presence of microbleeds, defined by small, well demarcated, hypointense, rounded lesions. In $\mathrm{AD}$, the prevalence of microbleeds is of 15 to $30 \%$, predominantly in the cortex, and should be due to frequent association between $\mathrm{AD}$ and cerebral amyloid angiopathy (CAA) (Cordonnier and van der Flier 2011).

\section{MRI based whole brain techniques}

Up to recently, the focus was made on the medial temporal structures, which are known to be an early site of tau pathology. With the development of automatic techniques such as MRI based whole brain volumetric and cortical thickness techniques, there is growing evidence of an early involvement of the neocortex. 


\section{Voxel-Based Morphometry (VBM)}

VBM is an automatic technique (implemented for instance in the SPM software ${ }^{1}$ ) exploring morphological differences across the whole-brain. It analyzes local differences in tissue concentration using univariate statistical tests performed at each voxel of images which have been previously segmented and aligned to a common space.

VBM studies have shown an early neocortical involvement with reduced grey matter in the posterior associate cortex and in the medial temporal lobe among MCI patients (Karas et al. 2004)(Hämäläinen et al. 2007). A greater GM loss in the medial temporal structures, in the posterior cingulate cortex and in the precuneus is thought to be predictive of conversion to AD dementia (Chételat et al. 2005). In addition, VBM could anticipate the rate of progression of AD. Patients with mild AD who will have a faster cognitive decline at 3 years already had more extensive cortical atrophy than patients with slower decline, especially in the medial occipitoparietal areas including the precuneus, which was not yet detected by clinical and neuropsychological assessment (Kinkingnéhun et al. 2008).

However, the interpretation of the differences obtained with VBM may prove difficult and it is not always possible to distinguish between local volume and shape changes. VBM is a method for group analysis. However, it is possible to use voxel-based measures as features for individual classification (Vemuri et al, 2008; Cuingnet et al, 2011).

\section{Cortical thickness}

Cortical thickness may provide a more direct index of gray matter atrophy than VBM and thus allow a better distinction between volume and shape changes. Fully automatic approaches (implemented for instance in the Freesurfer ${ }^{2}$ software) provide local measures of thickness at each point of the cortical surface. Therefore, thinning patterns can then be analyzed by performing univariate tests in each of these points.

Cortical thickness studies have shown that the medial temporal region, as well as the parietal association cortex and the prefrontal cortex, are significantly thinner in MCI patients (Singh et al. 2006), and longitudinal studies suggest that temporal (medial and inferior cortex, temporal pole) and parietal (superior parietal lobule) thinning allow differentiating the progressors from the non-progressors to Alzheimer's dementia (Bakkour et al. 2009).

Automatic classifiers based on cortical thickness or voxel-based measures have been designed to automatically distinguish individual patients. Their sensitivity and specificity

\footnotetext{
${ }^{1}$ http://www.fil.ion.ucl.ac.uk/spm/

${ }^{2}$ https://surfer.nmr.mgh.harvard.edu/
} 
reaches up to $80-95 \%$ for AD patients but is lower at the MCI stage (Cuingnet et al. 2011).

\section{Sulcal analysis}

A new method to measure morphological changes is based on the characteristics of cortical sulci. In particular, increased sulcal span is thought to be indicative of atrophy. An automatic approach (implemented in the Morphologist software of the Brainvisa platform ${ }^{3}$ ) is available to automatically segment and label up to 60 sulci per hemisphere. Conversely to VBM or cortical thickness techniques, it is not dependent on gray/white contrast that can be altered with age. Furthermore, this technique does not rely on analysis in a common stereotaxic space (i.e. it does not assume point-to-point correspondences across subjects) but instead provides an individual model of cortical anatomy.

Changes in the parietal occipital fissure and in the intraparietal sulcus are found in patients with Alzheimer disease and MCI (Reiner et al. 2012), which is largely consistent with results obtained with other techniques. Furthermore, a recent longitudinal study indicates that sulcal measures (width of the superior temporal, superior frontal sulcus and the sylvian fissure) could predict the conversion of MCI patients (Liu et al. 2013). Further studies are necessary to establish the sensitivity and predictive power of these measures.

\section{Diffusion Tensor Imaging (DTI)}

DTI provides quantitative information about the orientation and the integrity of white matter tracts by measuring the diffusion of water molecules in the brain tissue. Diffusivity of water depends on the presence of microscopic structural barriers in tissue that alters the random motion of water molecules. Pathologic disruption of cell membranes, loss of myelin and axonal alteration decrease the movement restriction of water and therefore increases the mean diffusivity (MD). In amnestic MCI patients, two meta analyses have shown that MD is increased in various regions with the largest effect size in the hippocampus (Sexton et al. 2011; Clerx et al. 2012). Furthermore, hippocampal MD is predictive of conversion to AD (Douaud et al. 2013). DTI also allows measuring fractional anisotropy (FA) corresponding to the degree of directionality of the diffusion along the axons of the WM. The loss of tract integrity decreases the FA at the MCI stage, mostly in the hippocampal, parahippocampal and cingular regions (Sexton et al. 2011; Clerx et al. 2012). However, DTI measures require further validation to be established as diagnostic biomarkers.

\footnotetext{
${ }^{3}$ http://brainvisa.info/
} 


\section{Functional magnetic resonance imaging (fMRI)}

fMRI can be used to study brain activity through the measure of the BOLD contrast (Blood oxygen level dependent) which reflects neuronal activation. It allows investigating functional intrinsic connectivity of large-scale neural networks, at rest or during cognitive tasks. Here, we focus on resting-state fMRI.

At rest, fMRI defines spatially distinct areas of the brain having synchronous BOLD fluctuations, which include the «default mode network » (DMN). This pattern of functional connectivity is active during resting periods and suspended during specific goal-directed behaviors (Raichle et al. 2001) and is altered in AD patients (Greicius et al. 2004). Several studies showed that the DMN is altered among patients with aMCI in the anterior medial frontal cortex and in the cingulate cortex compared to controls (Rombouts et al. 2005). A recent study showed high sensitivity and specificity for differentiating MCI and cognitively normal individuals, but the study included a small sample of patients (Chen et al. 2011). Furthermore, altered indices were significantly correlated with the results of cognitive tests (Chen et al. 2011). Finally, MCI subjects with disrupted connectivity in the DMN are shown to be at higher risk of converting to dementia within 4 years (Petrella et al. 2011). Further studies on larger groups of patients are necessary to establish the diagnostic accuracy of these measures.

\section{Conclusion}

Anatomical MRI markers support earlier diagnosis and measurement of progression. Medial temporal lobe atrophy measured with high-resolution MRI is correlated with both tau deposition and neuropsychological deficits. MRI measures of hippocampal atrophy are considered as a marker of neuronal injury in the new criteria of MCI due to AD (Albert et al. 2011). Whole-brain techniques that assess the overall pattern of atrophy without regional prior, provide complementary information which could improve early diagnosis. Finally, MRI provides new outcomes in clinical trials of potential disease-modifying therapies.

DTI and resting-state fMRI may provide additional markers of neuronal injury but require further validation. Moreover, measures may be difficult to compare across different imaging centers. An important effort of standardization is thus necessary for establishing these new measures as biomarkers in multicenter studies. 


\section{Disclosure}

OC reports having received lecture fees from Lundbeck, consulting fees from Guerbet and no other biomedical financial interests or any potential conflicts of interest. LH reports no biomedical financial interests or any potential conflicts of interest. MS reports having received lecture fees from Novartis and Lundbeck and no other biomedical financial interests or any potential conflicts of interest.

\section{References}

Albert MS, DeKosky ST, Dickson D, Dubois B, Feldman HH, Fox NC, et al. The diagnosis of mild cognitive impairment due to Alzheimer’s disease: recommendations from the National Institute on Aging-Alzheimer's Association workgroups on diagnostic guidelines for Alzheimer’s disease. Alzheimers Dement. 2011 May;7(3):270-9.

Bakkour A, Morris JC, Dickerson BC. The cortical signature of prodromal AD: regional thinning predicts mild AD dementia. Neurology. 2009 Mar 24;72(12):1048-55.

Chen G, Ward BD, Xie C, Li W, Wu Z, Jones JL, et al. Classification of Alzheimer disease, mild cognitive impairment, and normal cognitive status with large-scale network analysis based on resting-state functional MR imaging. Radiology. 2011 Apr;259(1):213-21.

Chételat G, Landeau B, Eustache F, Mézenge F, Viader F, de la Sayette V, et al. Using voxel-based morphometry to map the structural changes associated with rapid conversion in MCI: a longitudinal MRI study. Neuroimage. 2005 Oct 1;27(4):934-46.

Clerx L, Visser PJ, Verhey F, Aalten P. New MRI markers for Alzheimer’s disease: a meta-analysis of diffusion tensor imaging and a comparison with medial temporal lobe measurements. J. Alzheimers Dis. 2012;29(2):405-29.

Colliot O, Chételat G, Chupin M, Desgranges B, Magnin B, Benali H, et al. Discrimination between Alzheimer disease, mild cognitive impairment, and normal aging by using automated segmentation of the hippocampus. Radiology. 2008 Jul;248(1):194-201. 
Cordonnier C, van der Flier WM. Brain microbleeds and Alzheimer’s disease: innocent observation or key player? Brain. 2011 Feb;134(Pt 2):335-44.

Cuingnet R, Gerardin E, Tessieras J, Auzias G, Lehéricy S, Habert M-O, et al. Automatic classification of patients with Alzheimer's disease from structural MRI: a comparison of ten methods using the ADNI database. Neuroimage. 2011 May 15;56(2):766-81.

Douaud G, Menke RAL, Gass A, Monsch AU, Rao A, Whitcher B, et al. Brain microstructure reveals early abnormalities more than two years prior to clinical progression from mild cognitive impairment to Alzheimer’s disease. J. Neurosci. 2013 Jan 30;33(5):2147-55.

Gerardin E, Chételat G, Chupin M, Cuingnet R, Desgranges B, Kim H-S, et al. Multidimensional classification of hippocampal shape features discriminates Alzheimer's disease and mild cognitive impairment from normal aging. Neuroimage. 2009 Oct 1;47(4):1476-86.

Greicius MD, Srivastava G, Reiss AL, Menon V. Default-mode network activity distinguishes Alzheimer's disease from healthy aging: evidence from functional MRI. Proc. Natl. Acad. Sci. U.S.A. 2004 Mar 30;101(13):4637-42.

Hämäläinen A, Tervo S, Grau-Olivares M, Niskanen E, Pennanen C, Huuskonen J, et al. Voxel-based morphometry to detect brain atrophy in progressive mild cognitive impairment. Neuroimage. 2007 Oct 1;37(4):1122-31.

Hashimoto M, Kitagaki H, Imamura T, Hirono N, Shimomura T, Kazui H, et al. Medial temporal and whole-brain atrophy in dementia with Lewy bodies: a volumetric MRI study. Neurology. 1998 Aug;51(2):357-62.

Jack CR Jr, Petersen RC, Xu Y, O’Brien PC, Smith GE, Ivnik RJ, et al. Rates of hippocampal atrophy correlate with change in clinical status in aging and AD. Neurology. 2000 Aug 22;55(4):484-9. 
Karas GB, Scheltens P, Rombouts SARB, Visser PJ, van Schijndel RA, Fox NC, et al. Global and local gray matter loss in mild cognitive impairment and Alzheimer's disease. Neuroimage. 2004 Oct;23(2):708-16.

Kinkingnéhun S, Sarazin M, Lehéricy S, Guichart-Gomez E, Hergueta T, Dubois B. VBM anticipates the rate of progression of Alzheimer disease: a 3-year longitudinal study. Neurology. 2008 Jun 3;70(23):2201-11.

Laakso MP, Partanen K, Riekkinen P, Lehtovirta M, Helkala EL, Hallikainen M, et al. Hippocampal volumes in Alzheimer's disease, Parkinson’s disease with and without dementia, and in vascular dementia: An MRI study. Neurology. 1996 Mar;46(3):678-81.

Lehéricy S, Marjanska M, Mesrob L, Sarazin M, Kinkingnehun S. Magnetic resonance imaging of Alzheimer’s disease. Eur Radiol. 2007 Feb;17(2):347-62.

Liu T, Sachdev PS, Lipnicki DM, Jiang J, Cui Y, Kochan NA, et al. Longitudinal changes in sulcal morphology associated with late-life aging and MCI. Neuroimage. $2013 \mathrm{Jul}$ $1 ; 74: 337-42$.

Mueller SG, Chao LL, Berman B, Weiner MW. Evidence for functional specialization of hippocampal subfields detected by MR subfield volumetry on high resolution images at $4 \mathrm{~T}$. Neuroimage. 2011 Jun 1;56(3):851-7.

Petrella JR, Sheldon FC, Prince SE, Calhoun VD, Doraiswamy PM. Default mode network connectivity in stable vs progressive mild cognitive impairment. Neurology. 2011 Feb 8;76(6):511-7.

Raichle ME, MacLeod AM, Snyder AZ, Powers WJ, Gusnard DA, Shulman GL. A default mode of brain function. Proc. Natl. Acad. Sci. U.S.A. 2001 Jan 16;98(2):676-82.

Reiner P, Jouvent E, Duchesnay E, Cuingnet R, Mangin J-F, Chabriat H, et al. Sulcal span in Azheimer's disease, amnestic mild cognitive impairment, and healthy controls. J. Alzheimers Dis. 2012;29(3):605-13. 
Rombouts SARB, Barkhof F, Goekoop R, Stam CJ, Scheltens P. Altered resting state networks in mild cognitive impairment and mild Alzheimer's disease: an fMRI study. Hum Brain Mapp. 2005 Dec;26(4):231-9.

Sarazin M, Chauviré V, Gerardin E, Colliot O, Kinkingnéhun S, de Souza LC, et al. The amnestic syndrome of hippocampal type in Alzheimer's disease: an MRI study. J. Alzheimers Dis. 2010;22(1):285-94.

Scheltens P, Fox N, Barkhof F, De Carli C. Structural magnetic resonance imaging in the practical assessment of dementia: beyond exclusion. Lancet Neurol. 2002 May;1(1):13-21.

Sexton CE, Kalu UG, Filippini N, Mackay CE, Ebmeier KP. A meta-analysis of diffusion tensor imaging in mild cognitive impairment and Alzheimer's disease. Neurobiol. Aging. 2011 Dec;32(12):2322.e5-18.

Singh V, Chertkow H, Lerch JP, Evans AC, Dorr AE, Kabani NJ. Spatial patterns of cortical thinning in mild cognitive impairment and Alzheimer’s disease. Brain. 2006 Nov;129(Pt 11):2885-93.

De Souza LC, Chupin M, Bertoux M, Lehéricy S, Dubois B, Lamari F, et al. Is hippocampal volume a good marker to differentiate Alzheimer's disease from frontotemporal dementia? J. Alzheimers Dis. 2013 Jan 1;36(1):57-66.

De Souza LC, Chupin M, Lamari F, Jardel C, Leclercq D, Colliot O, et al. CSF tau markers are correlated with hippocampal volume in Alzheimer’s disease. Neurobiol. Aging. 2012 Jul;33(7):1253-7.

Vemuri P, Whitwell JL, Kantarci K, Josephs KA, Parisi JE, Shiung MS, et al. Antemortem MRI based STructural Abnormality iNDex (STAND)-scores correlate with postmortem Braak neurofibrillary tangle stage. Neuroimage. 2008 Aug 15;42(2):559-67.

Vemuri P, Wiste HJ, Weigand SD, Shaw LM, Trojanowski JQ, Weiner MW, et al. MRI and CSF biomarkers in normal, MCI, and AD subjects: diagnostic discrimination and 
cognitive correlations. Neurology. 2009 Jul 28;73(4):287-93. 